\title{
Note on Relation between Double Laplace Transform and Double Differential Transform
}

\author{
Hassan Eltayeb \\ Mathematics Department, College of Science, King Saud University, P.O. Box 2455, Riyadh 11451, Saudi Arabia \\ Correspondence should be addressed to Hassan Eltayeb; hgadain@ksu.edu.sa \\ Received 12 May 2013; Accepted 8 June 2013 \\ Academic Editor: Bessem Samet \\ Copyright (C) 2013 Hassan Eltayeb. This is an open access article distributed under the Creative Commons Attribution License, \\ which permits unrestricted use, distribution, and reproduction in any medium, provided the original work is properly cited.
}

Double differential transform method has been employed to compute double Laplace transform. To illustrate the method, four examples of different forms have been prepared.

\section{Introduction}

The concept of the DTM was first proposed by Zhou [1], who solved linear and nonlinear problems in electrical circuit problems. Chen and Ho [2] developed this method for partial differential equations and applied it to the system of differential equations. During the recent years, many authors have used this method for solving various types of equations. For example, this method has been used for differential algebraic equations [3], partial differential equations $[4,5]$, fractional differential equations [6], Volterra integral equations [7], and difference equations [8]. The main goal of this paper is to extend the study of single Laplace transform by using differential transform (see [9]) and to compute double Laplace transform by means of double differential transform method. As we know, the standard derivation of Laplace transforms inherits an improper integration which may, in certain cases, not be analytically tractable. However, in contrast, the proposed straightforward approach merely requires easy differentiations and algebraic operations. Three examples are proposed.

The one-dimensional differential transform of the function $u(x)$ is defined by the following formula:

$$
U(k)=\frac{1}{k !}\left[\frac{d^{k} u(x)}{d x^{k}}\right]_{x=0},
$$

where $u(x)$ and $U(k)$ are the original and transform functions, respectively. The inverse differential transform of $U(k)$ is specified as follows:

$$
u(x)=\sum_{k=0}^{\infty}\left[U(k) x^{k}\right] .
$$

Consider an analytical function $u(x, t)$ of two variables; then this function can be represented as a series in $\left(x_{0}, t_{0}\right) \in D$ using differential transform

$$
U(m, n)=\frac{1}{m ! n !}\left[\frac{\partial^{m+n} u(x, t)}{\partial x^{m} \partial t^{n}}\right]_{x=x_{0}, t=t_{0}}
$$

and inverse double differential transform

$$
u(x, t)=\sum_{m=0}^{\infty} \sum_{n=0}^{\infty} \frac{1}{m ! n !}\left[\frac{\partial^{m+n} u(x, t)}{\partial x^{m} \partial t^{n}}\right]_{x=0, t=0} .
$$

From the definition of double Laplace transform, we can write

$$
\begin{aligned}
L_{x} L_{t}[f(x, t)] & =F(p, s) \\
& =\left[\int_{x_{0}}^{x} e^{-p x}\left[\int_{t_{0}}^{t} e^{-s t} f(x, t) d t\right]_{t_{0}=0}^{t=\infty} d x\right]_{x_{0}=0}^{x=\infty},
\end{aligned}
$$

where $x, t>0$ and $p, s$ is a complex value. 
On using double direct and inverse differential transform with respect to $x$ and $t$ for both sides of the previous,

$$
\begin{aligned}
F(p, s) & \\
= & D T_{p}^{-1} D T_{s}^{-1} \\
& \times\left\{D T_{x} D T_{t}\right. \\
& \left.\times\left\{\left[\int_{x_{0}}^{x} e^{-p x}\left[\int_{t_{0}}^{t} e^{-s t} f(x, t) d t\right]_{t_{0}=0}^{t=\infty} d x\right]_{x_{0}=0}^{x=\infty}\right\}\right\}, \\
F(p, s) & {\left[\left[D T_{p}^{-1} D T_{s}^{-1}\right.\right.} \\
\times & \left.\left.\times\left\{D T_{x} \int_{x_{0}} e^{-p x}\left\{D T_{t} \int_{t_{0}}^{t} e^{-s t} f(x, t) d t\right\} d x\right\}\right]_{t_{0}=0}^{t=\infty}\right]_{x_{0}=0}^{x=\infty} .
\end{aligned}
$$

Lemma 1. Let $m$ and $n$ be a finite positive integers such that $m, n \geq 1$ and $s, p>0, s, p \in R^{2}$; then

$$
\lim _{\substack{x \rightarrow \infty \\ t \rightarrow \infty}} \sum_{k=1}^{\infty} \sum_{r=1}^{\infty}\left[\frac{k^{m} r^{n}}{k ! r !}(-s t)^{k}(-p x)^{r}\right]=0 .
$$

Proof. By using mathematical induction, letting $m, n=1$, we have

$$
\begin{aligned}
\left(\sum_{k=1}^{\infty} \frac{k}{k !}(-s t)^{k}\right)\left(\sum_{r=1}^{\infty} \frac{r}{r !}(-p x)^{r}\right) \\
=\left(\sum_{k=1}^{\infty} \frac{1}{(k-1) !}(-s t)^{k}\right)\left(\sum_{r=1}^{\infty} \frac{1}{(r-1) !}(-p x)^{r}\right) \\
=\left(\sum_{k=0}^{\infty} \frac{1}{k !}(-s t)^{k+1}\right)\left(\sum_{r=0}^{\infty} \frac{1}{r !}(-p x)^{r+1}\right) \\
=\left((-s t) \sum_{k=0}^{\infty} \frac{1}{k !}(-s t)^{k}\right)\left((-p x) \sum_{r=0}^{\infty} \frac{1}{r !}(-p x)^{r}\right) \\
=(-s t) e^{-s t}(-p x) e^{-p x} .
\end{aligned}
$$

Then $\quad \lim _{\substack{x \rightarrow \infty \\ t \rightarrow \infty}} \sum_{k=1}^{\infty} \sum_{r=1}^{\infty}\left[(k r / k ! r !)(-s t)^{k}(-p x)^{r}\right]=$ $\lim _{x \rightarrow \infty}(-s t) e^{-s t}(-p x) e^{-p x}=0$; since $\left(\sum_{k=1}^{\infty}(k / k !)(-s t)^{k}\right)$ $\left(\sum_{r=1}^{\infty}(r / r !)(-p x)^{r}\right)=(-s t) e^{-s t}(-p x) e^{-p x}$ we conclude that

$$
\lim _{\substack{x \rightarrow \infty \\ t \rightarrow \infty}}(-s t)(-p x)\left(\sum_{k=1}^{\infty} \frac{k}{k !}(-s t)^{k}\right)\left(\sum_{r=1}^{\infty} \frac{r}{r !}(-p x)^{r}\right)=0,
$$

for $m, n=2$,

$$
\begin{aligned}
\left(\sum_{k=1}^{\infty} \frac{k^{2}}{k !}(-s t)^{k}\right)\left(\sum_{r=1}^{\infty} \frac{r^{2}}{r !}(-p x)^{r}\right) \\
=\left(\sum_{k=1}^{\infty} \frac{k}{(k-1) !}(-s t)^{k}\right)\left(\sum_{r=1}^{\infty} \frac{r}{(r-1) !}(-p x)^{r}\right) \\
=\left(\sum_{k=0}^{\infty} \frac{k+1}{k !}(-s t)^{k+1}\right)\left(\sum_{r=0}^{\infty} \frac{r+1}{r !}(-p x)^{r+1}\right) \\
=(-s t)\left(\sum_{k=0}^{\infty} \frac{k}{k !}(-s t)^{k}+\sum_{k=0}^{\infty} \frac{(-s t)^{k}}{k !}\right) \\
\quad \times(-p x)\left(\sum_{r=0}^{\infty} \frac{r}{r !}(-p x)^{r}+\sum_{r=0}^{\infty} \frac{(-p x)^{r}}{r !}\right) \\
=(-s t)\left[(-s t) e^{-s t}+e^{-s t}\right] \\
\quad \times(-p x)\left[(-p x) e^{-p x}+e^{-p x}\right] .
\end{aligned}
$$

Consequently

$$
\begin{aligned}
\lim _{\substack{x \rightarrow \infty \\
t \rightarrow \infty}}\left(\sum_{k=1}^{\infty} \frac{k^{2}}{k !}(-s t)^{k}\right)\left(\sum_{r=1}^{\infty} \frac{r^{2}}{r !}(-p x)^{r}\right) \\
=\lim _{\substack{x \rightarrow \infty \\
t \rightarrow \infty}}(-s t)\left[(-s t) e^{-s t}+e^{-s t}\right] \\
\quad \times(-p x)\left[(-p x) e^{-p x}+e^{-p x}\right]=0 .
\end{aligned}
$$

Similarly

$$
\begin{gathered}
\lim _{\substack{x \rightarrow \infty \\
t \rightarrow \infty}}(-s t)(-p x)\left(\sum_{k=1}^{\infty} \frac{k^{2}}{k !}(-s t)^{k}\right)\left(\sum_{r=1}^{\infty} \frac{r^{2}}{r !}(-p x)^{r}\right) \\
=\lim _{\substack{x \rightarrow \infty \\
t \rightarrow \infty}}(-s t)^{2}\left[(-s t) e^{-s t}+e^{-s t}\right] \\
\quad \times(-p x)^{2}\left[(-p x) e^{-p x}+e^{-p x}\right]=0 .
\end{gathered}
$$

Assume that, for $m, n=1$ to $i, j$, it holds that

$$
\lim _{\substack{x \rightarrow \infty \\ t \rightarrow \infty}} \sum_{k=1}^{\infty} \sum_{r=1}^{\infty}\left[\frac{k^{m} r^{n}}{k ! r !}(-s t)^{k}(-p x)^{r}\right]=0,
$$

and also

$$
\begin{gathered}
\lim _{\substack{x \rightarrow \infty \\
t \rightarrow \infty}}(-s t)(-p x)\left(\sum_{k=1}^{\infty} \frac{k^{m}}{k !}(-s t)^{k}\right) \\
\times\left(\sum_{r=1}^{\infty} \frac{r^{n}}{r !}(-p x)^{r}\right)=0 .
\end{gathered}
$$


Now, we are going to prove that

$$
\begin{aligned}
& \lim _{\substack{x \rightarrow \infty \\
t \rightarrow \infty}} \sum_{k=1}^{\infty} \sum_{r=1}^{\infty}\left[\frac{k^{m+1} r^{n+1}}{k ! r !}(-s t)^{k}(-p x)^{r}\right]=0, \\
& \sum_{k=1}^{\infty} \sum_{r=1}^{\infty}\left[\frac{k^{m+1} r^{n+1}}{k ! r !}(-s t)^{k}(-p x)^{r}\right] \\
&=\sum_{k=1}^{\infty} \sum_{r=1}^{\infty}\left[\frac{k^{m} r^{n}}{(k-1) !(r-1) !}(-s t)^{k}(-p x)^{r}\right] \\
&=(-s t)(-p x) \sum_{k=0}^{\infty} \sum_{r=0}^{\infty}\left[\frac{(k+1)^{m}(r+1)^{n}}{k ! r !}(-s t)^{k}(-p x)^{r}\right] .
\end{aligned}
$$

By using the definition of polynomial, we have

$$
\begin{aligned}
& (-s t) \sum_{k=0}^{\infty}\left[\frac{(k+1)^{m}}{k !}(-s t)^{k}\right](-p x) \sum_{r=0}^{\infty}\left[\frac{(r+1)^{n}}{r !}(-p x)^{r}\right] \\
& =(-s t) \sum_{k=0}^{\infty}\left[\frac{\left(1+\left(\begin{array}{c}
m \\
1
\end{array}\right) k+\left(\begin{array}{c}
m \\
2
\end{array}\right) k^{2}+\cdots+k^{m}\right)}{k !}(-s t)^{k}\right] \\
& \times(-p x) \sum_{r=0}^{\infty}\left[\frac{\left(1+\left(\begin{array}{c}
n \\
1
\end{array}\right) r+\left(\begin{array}{c}
n \\
2
\end{array}\right) r^{2}+\cdots+r^{n}\right)}{r !}(-p x)^{r}\right] \\
& =\left((-s t) \sum_{k=0}^{\infty} \frac{(-s t)^{k}}{k !}+\left(\begin{array}{c}
m \\
1
\end{array}\right)(-s t) \sum_{k=0}^{\infty} \frac{k(-s t)^{k}}{k !}\right. \\
& +\left(\begin{array}{c}
m \\
2
\end{array}\right)(-s t) \sum_{k=0}^{\infty} \frac{k^{2}(-s t)^{k}}{k !} \\
& \left.+\cdots+(-s t) \sum_{k=0}^{\infty} \frac{k^{m}(-s t)^{k}}{k !}\right) \\
& \times\left((-p x) \sum_{r=0}^{\infty} \frac{(-p x)^{r}}{r !}+\left(\begin{array}{c}
n \\
1
\end{array}\right)(-p x) \sum_{r=0}^{\infty} \frac{r(-p x)^{r}}{r !}\right. \\
& +\left(\begin{array}{l}
n \\
2
\end{array}\right)(-p x) \sum_{r=0}^{\infty} \frac{r^{2}(-p x)^{r}}{r !} \\
& \left.+\cdots+(-p x) \sum_{r=0}^{\infty} \frac{r^{n}(-p x)^{r}}{r !}\right) \text {. }
\end{aligned}
$$

Thus

$$
\lim _{\substack{x \rightarrow \infty \\ t \rightarrow \infty}} \sum_{k=1}^{\infty} \sum_{r=1}^{\infty}\left[\frac{k^{m+1} r^{n+1}}{k ! r !}(-s t)^{k}(-p x)^{r}\right]=0
$$

\section{Relation between Double Laplace and Differential Transforms}

In this section, we compute the double Laplace transform by means of double differential transform by proposing some examples as follows.
Example 2. Double Laplace transform of function $e^{a x+b t}$; consider

$$
\begin{aligned}
& L_{x} L_{t}\left\{e^{a x+b t}\right\} \\
& =\left[D T_{p}^{-1} D T_{s}^{-1}\right. \\
& \quad \times\left\{D T_{x} D T_{t}\right. \\
& \left.\left.\quad \times\left\{\int_{y_{0}}^{y} e^{a x-p x} d x \int_{\beta_{0}}^{\beta} e^{b t-s t} d t\right\}\right\}\right]_{y_{0}=0, \beta_{0}=0}^{y=\infty, \beta=\infty}
\end{aligned}
$$$$
\begin{gathered}
=\left[D T_{p}^{-1}\left\{\frac{(-p+a)^{r-1}}{r(r-1) !}\right\}\right]_{y_{0}=0}^{y=\infty} \\
\times\left[D T_{s}^{-1}\left\{\frac{(-s+b)^{k-1}}{k(k-1) !}\right\}\right]_{\beta_{0}=0}^{\beta=\infty}
\end{gathered}
$$$$
=\left[\sum_{r=0}^{\infty}\left\{\frac{(-p+a)^{r-1} y^{r}}{r(r-1) !}\right\}\right]_{y_{0}=0}^{y=\infty}
$$$$
\times\left[\sum_{k=0}^{\infty}\left\{\frac{(-s+b)^{k-1} \beta^{k}}{k(k-1) !}\right\}\right]_{\beta_{0}=0}^{\beta=\infty}
$$$$
=\frac{1}{(a-p)}\left[\sum_{r=0}^{\infty}\left\{\frac{(-p+a)^{r} y^{r}}{r !}\right\}\right]_{y_{0}=0}^{y=\infty}
$$$$
\times \frac{1}{(b-s)}\left[\sum_{k=0}^{\infty}\left\{\frac{(-s+b)^{k} \beta^{k}}{k !}\right\}\right]_{\beta_{0}=0}^{\beta=\infty}
$$$$
=\frac{1}{(a-p)}\left[e^{(a-p) y}\right]_{y_{0}=0}^{y=\infty} \frac{1}{(b-s)}\left[e^{(b-s) \beta}\right]_{\beta_{0}=0}^{\beta=\infty}
$$$$
=\frac{1}{(p-a)(s-b)}, \quad p>a, s>b \text {. }
$$

In the next example, we apply double differential transform to compute double Laplace transform as follows.

Example 3. If we consider the function $x^{m} t^{n}$, then double Laplace transform is given by

$$
\begin{aligned}
L_{x} L_{t} & \left\{x^{m} t^{n} ; p, s\right\} \\
= & {\left[D T_{p}^{-1} D T_{s}^{-1}\right.} \\
& \left.\times\left\{D T_{x} D T_{t}\left\{\int_{y_{0}}^{y} x^{m} e^{-p x} d x \int_{\beta_{0}}^{\beta} t^{n} e^{-s t} d t\right\}\right\}\right]_{y_{0}=0, \beta_{0}=0}^{y=\infty, \beta=\infty}
\end{aligned}
$$




$$
\begin{gathered}
=\left[\sum_{r=0}^{\infty}\left\{D T_{x}\left\{\int_{y_{0}}^{y} x^{m} e^{-p x} d x\right\} y^{r}\right\}\right]_{y_{0}=0}^{y=\infty} \\
\times\left[\sum_{k=0}^{\infty}\left\{D T_{t}\left\{\int_{\beta_{0}}^{\beta} t^{n} e^{-s t} d t\right\} \beta^{k}\right\}\right]_{\beta_{0}=0}^{\beta=\infty} .
\end{gathered}
$$

From the properties of double differential transform, we have

$$
\begin{aligned}
& L_{x} L_{t}\left\{x^{m} t^{n}\right\} \\
& =0+\left[\sum_{r=0}^{\infty}\left\{\sum_{l=0}^{r-1}\left\{\frac{\delta(r-m-l-1)}{r} \frac{(-p)^{l}}{l !}\right\} y^{r}\right\}\right]_{y_{0}=0}^{y=\infty} \\
& \times\left[\sum_{k=0}^{\infty}\left\{\sum_{c=0}^{k-1}\left\{\frac{\delta(k-n-c-1)}{k} \frac{(-s)^{c}}{c !}\right\} \beta^{k}\right\}\right]_{\beta_{0}=0}^{\beta=\infty} .
\end{aligned}
$$

On using definition of Kronecker delta function forces, we have

$$
\begin{aligned}
L_{x} L_{t} & \left\{x^{m} t^{n}\right\} \\
= & {\left[\sum_{r=0}^{\infty}\left\{\frac{1}{r} \frac{(-p)^{r-1-m}}{(r-1-m) !}\right\} y^{r}\right]_{y_{0}=0}^{y=\infty} } \\
& \times\left[\sum_{k=0}^{\infty}\left\{\frac{1}{k} \frac{(-s)^{k-1-n}}{(k-1-n) !}\right\} \beta^{k}\right]_{\beta_{0}=0}^{\beta=\infty} \\
= & {\left[\frac{(-1)^{m+1}}{p^{m+1}}\right.} \\
& \left.\times \sum_{r=1}^{\infty}\left\{\frac{(r-1)(r-2) \cdots(r-m)(-p)^{r}}{r !} y^{r}\right\}\right]_{y_{0}=0}^{y=\infty} \\
& \times\left[\frac{(-1)^{n+1}}{s^{n+1}}\right. \\
& \left.\times \sum_{k=0}^{\infty}\left\{\frac{(k-1)(k-2) \cdots(k-n)(-s)^{k}}{k !} \beta^{k}\right\}\right]_{\beta_{0}=0}^{\beta=\infty} .
\end{aligned}
$$

From the previous equation, we have

$$
\begin{aligned}
& L_{x} L_{t}\left\{x^{m} t^{n} ; p, s\right\} \\
& =\frac{(-1)^{m+1}}{p^{m+1}}\left(\left[\sum_{r=1}^{\infty}\left\{\frac{(-1)(-2) \cdots(-m)(-p)^{r}}{r !} y^{r}\right\}\right]_{y_{0}=0}^{y=\infty}\right. \\
& \left.+\sum_{i=1}^{m} a_{i} \lim _{y \rightarrow \infty} \sum_{r=1}^{\infty}\left\{\frac{r^{i}}{r !} y^{r}\right\}-0\right)
\end{aligned}
$$

$$
\begin{gathered}
\times \frac{(-1)^{n+1}}{s^{n+1}}\left(\left[\sum_{k=1}^{\infty}\left\{\frac{(-1)(-2) \cdots(-n)(-s)^{k}}{k !} \beta^{k}\right\}\right]_{\beta_{0}=0}^{\beta=\infty}\right. \\
\left.+\sum_{j=1}^{n} b_{j} \lim _{\beta \rightarrow \infty} \sum_{k=1}^{\infty}\left\{\frac{k^{j}}{k !} \beta^{k}\right\}-0\right),
\end{gathered}
$$

where $a_{i}$ and $b_{j}$ are constant coefficients of the polynomials generated by $(r-1)(r-2) \cdots(r-m)$ and $(k-1)(k-2) \cdots(k-n)$, respectively. According to the lemma, the last summations of (23), $\lim _{y \rightarrow \infty} \sum_{r=1}^{\infty}\left\{\left(r^{i} / r !\right) y^{r}\right\}$, and $\lim _{\beta \rightarrow \infty} \sum_{k=1}^{\infty}\left\{\left(k^{j} / k !\right) \beta^{k}\right\}$ are zeros, such that

$$
\begin{aligned}
& L_{x} L_{t}\left\{x^{m} t^{n}\right\} \\
& =\frac{(-1)^{m+1}}{p^{m+1}}\left[\sum_{r=1}^{\infty}\left\{\frac{(-1)(-2) \cdots(-m)(-p)^{r}}{r !} y^{r}\right\}\right]_{y_{0}=0}^{y=\infty} \\
& \times \frac{(-1)^{n+1}}{s^{n+1}}\left[\sum_{k=1}^{\infty}\left\{\frac{(-1)(-2) \cdots(-n)(-s)^{k}}{k !} \beta^{k}\right\}\right]_{\beta_{0}=0}^{\beta=\infty} \\
& =\frac{(-1)^{m+1} m !}{p^{m+1}}\left[\sum_{r=1}^{\infty}\left\{\frac{(-p)^{r}}{r !} y^{r}\right\}\right]_{y_{0}=0}^{y=\infty} \\
& \times \frac{(-1)^{n+1} n !}{s^{n+1}}\left[\sum_{k=1}^{\infty}\left\{\frac{(-s)^{k}}{k !} \beta^{k}\right\}\right]_{\beta_{0}=0}^{\beta=\infty} \\
& =\frac{-m !}{p^{m+1}}\left[\sum_{r=1}^{\infty}\left\{\frac{(-p)^{r}}{r !} y^{r}\right\}\right]_{y_{0}=0}^{y=\infty} \\
& \times \frac{-n !}{s^{n+1}}\left[\sum_{k=1}^{\infty}\left\{\frac{(-s)^{k}}{k !} \beta^{k}\right\}\right]_{\beta_{0}=0}^{\beta=\infty} \\
& =\frac{-m !}{p^{m+1}}\left[\left(e^{-p y}-1\right)\right]_{y_{0}=0}^{y=\infty} \\
& \times \frac{-n !}{s^{n+1}}\left[\left(e^{-s \beta}-1\right)\right]_{\beta_{0}=0}^{\beta=\infty} \\
& =\frac{m ! n !}{p^{m+1} s^{n+1}} .
\end{aligned}
$$

In the next example, we apply double integral transform as follows.

Example 4. If we consider the function $\sin (a x) \sin (b t)$, then double Laplace transform is given by

$$
\begin{aligned}
& L_{x} L_{t}\{\sin (a x) \sin (b t) ; p, s\} \\
&=\left[D T_{p}^{-1} D T_{s}^{-1}\right. \\
& \times\left\{D T_{x} D T_{t}\right.
\end{aligned}
$$




$$
\left.\left.\times\left\{\int_{y_{0}}^{y} \int_{\beta_{0}}^{\beta} \sin (a x) \sin (b t) e^{-p x} e^{-s t} d t d x\right\}\right\}\right]_{y_{0}=0, \beta_{0}=0}^{y=\infty, \beta=\infty},
$$

$L_{x} L_{t}\{\sin (a x) \sin (b t) ; p, s\}$

$$
\begin{aligned}
= & {\left[D T_{p}^{-1}\right.} \\
& \left.\times\left\{\sum_{l=0}^{r-1}\left\{\frac{a^{j}}{j !} \sin \left(\frac{j \pi}{2}\right) \frac{(-p)^{r-j-1} /(r-j-1) !}{r}\right\}\right\}\right]_{y_{0}=0}^{y=\infty} \\
& \times\left[D T_{s}^{-1}\right. \\
& \left.\times\left\{\sum_{i=0}^{k-1}\left\{\frac{b^{i}}{i !} \sin \left(\frac{i \pi}{2}\right) \frac{(-s)^{k-i-1} /(k-i-1) !}{k}\right\}\right\}\right]_{\beta_{0}=0}^{\beta=\infty}
\end{aligned}
$$

$L_{x} L_{t}\{\sin (a x) \sin (b t) ; p, s\}$

$$
=\left[\sum _ { r = 0 } ^ { \infty } \left\{\sum _ { l = 1 , 3 , 5 } ^ { r - 1 } \left\{\frac{a^{j}}{j !} \frac{(r-1)(r-2) \cdots(r-j)}{p^{j+1}}\right.\right.\right.
$$

$$
\left.\left.\left.\times(-1)^{(j-1) / 2-j-1}\right\} \frac{y^{r}(-p)^{r}}{r !}\right\}\right]_{y_{0}=0}^{y=\infty}
$$

$$
\times\left[\sum _ { k = 0 } ^ { \infty } \left\{\sum _ { i = 1 , 3 , 5 } ^ { k - 1 } \left\{\frac{b^{i}}{i !} \frac{(k-1)(k-2) \cdots(k-i)}{s^{i+1}}\right.\right.\right.
$$

$$
\left.\left.\left.\times(-1)^{(i-1) / 2-i-1}\right\} \frac{\beta^{k}(-s)^{k}}{k !}\right\}\right]_{\beta_{0}=0}^{\beta=\infty},
$$

$L_{x} L_{t}\{\sin (a x) \sin (b t) ; p, s\}$

$$
\begin{aligned}
& =\left[\sum _ { r = 0 } ^ { \infty } \left\{\sum _ { l = 1 , 3 , 5 } ^ { r - 1 } \left\{\frac{a^{j}}{j !} \frac{(r-1)(r-2) \cdots(r-j)}{p^{j+1}}\right.\right.\right. \\
& \left.\left.\left.\times(-1)^{(j+3) / 2}\right\} \frac{y^{r}(-p)^{r}}{r !}\right\}\right]_{y_{0}=0}^{y=\infty} \\
& \times\left[\sum _ { k = 0 } ^ { \infty } \left\{\sum _ { i = 1 , 3 , 5 } ^ { k - 1 } \left\{\frac{b^{i}}{i !} \frac{(k-1)(k-2) \cdots(k-i)}{s^{i+1}}\right.\right.\right. \\
& \left.\left.\left.\times(-1)^{(i+3) / 2}\right\} \frac{\beta^{k}(-s)^{k}}{k !}\right\}\right]_{\beta_{0}=0}^{\beta=\infty}
\end{aligned}
$$

By calculating the summation inside the bracket we have

$$
\begin{aligned}
L_{x} L_{t} & \{\sin (a x) \sin (b t) ; p, s\} \\
= & {\left[\sum _ { r = 0 } ^ { \infty } \left\{\frac{a}{p^{2}} \frac{(r-1)(-p y)^{r}}{r !}\right.\right.}
\end{aligned}
$$

$$
\begin{gathered}
-\frac{a^{3}(r-1)(r-2)(r-3)}{p^{4}} \frac{(-p y)^{r}}{r !} \\
\left.\left.+\frac{a^{5}(r-1)(r-2)(r-3)}{p^{6}} \frac{(-p y)^{r}}{r !}+\cdots\right\}\right]_{y_{0}=0}^{y=\infty} \\
\times\left[\sum _ { k = 0 } ^ { \infty } \left\{\frac{b}{s^{2}} \frac{(k-1)(-s \beta)^{k}}{k !}\right.\right. \\
-\frac{b^{3}(k-1)(k-2)(k-3)}{s^{4}} \frac{(-s \beta)^{k}}{k !} \\
\left.\left.+\frac{b^{5}(k-1)(k-2)(k-3)}{s^{6}} \frac{(-s \beta)^{k}}{k !}+\cdots\right\}\right]_{\beta_{0}=0}^{\beta=\infty} .
\end{gathered}
$$

From the previous lemma, we know that, for $m, n \geq 1$, $\lim _{y \rightarrow \infty} \sum_{r=1}^{\infty}\left\{\left(r^{m} / r !\right)(-p y)^{r}\right\}=0$, and $\lim _{\beta \rightarrow \infty} \sum_{k=1}^{\infty}$ $\left\{\left(k^{m} / k !\right)(-s \beta)^{k}\right\}=0$, we have the following form:

$$
\begin{aligned}
& L_{x} L_{t}\{\sin (a x) \sin (b t) ; p, s\} \\
& =\left[\sum _ { r = 0 } ^ { \infty } \left\{-\frac{a}{p^{2}} \frac{(-p y)^{r}}{r !}+\frac{a^{3}}{p^{4}} \frac{(-p y)^{r}}{r !}\right.\right. \\
& \left.\left.-\frac{a^{5}}{p^{6}} \frac{(-p y)^{r}}{r !}+\cdots\right\}\right]_{y_{0}=0}^{y=\infty} \\
& \times\left[\sum _ { k = 0 } ^ { \infty } \left\{-\frac{b}{s^{2}} \frac{(-s \beta)^{k}}{k !}+\frac{b^{3}}{s^{4}} \frac{(-s \beta)^{k}}{k !}\right.\right. \\
& \left.\left.-\frac{b^{5}}{s^{6}} \frac{(-s \beta)^{k}}{k !}+\cdots\right\}\right]_{\beta_{0}=0}^{\beta=\infty} \\
& =\left[\left(-\frac{a}{p^{2}}+\frac{a^{3}}{p^{4}}-\frac{a^{5}}{p^{6}}+\cdots\right) e^{-p y}\right]_{y_{0}=0}^{y=\infty} \\
& \times\left[\left(-\frac{b}{s^{2}}+\frac{b^{3}}{s^{4}}-\frac{b^{5}}{s^{6}}+\cdots\right) e^{-s \beta}\right]_{\beta_{0}=0}^{\beta=\infty} \\
& =\left(\frac{a}{p^{2}}-\frac{a^{3}}{p^{4}}+\frac{a^{5}}{p^{6}}+\cdots\right) \\
& \times\left(\frac{b}{s^{2}}-\frac{b^{3}}{s^{4}}+\frac{b^{5}}{s^{6}}+\cdots\right) .
\end{aligned}
$$

From the definition of infinite geometric series and the summation of the previous terms, we have

$$
\begin{aligned}
L_{x} L_{t} & \{\sin (a x) \sin (b t) ; p, s\} \\
= & \lim _{m \rightarrow \infty} \frac{a}{p^{2}} \frac{\left(1-\left(-a^{2} / p^{2}\right)^{m+1}\right)}{1-\left(-a^{2} / p^{2}\right)}
\end{aligned}
$$




$$
\begin{aligned}
& \times \lim _{n \rightarrow \infty} \frac{b}{s^{2}} \frac{\left(1-\left(-b^{2} / s^{2}\right)^{n+1}\right)}{1-\left(-b^{2} / s^{2}\right)} \\
= & \left(\frac{a / p^{2}}{1+a^{2} / p^{2}}\right)\left(\frac{b / s^{2}}{1+b^{2} / s^{2}}\right)=\frac{a b}{\left(p^{2}+a^{2}\right)\left(s^{2}+b^{2}\right)} .
\end{aligned}
$$

In the next example, we apply double differential transform to find double Laplace transform of the function $(\sin (x) / x)(\sin (t) / t)$ as follows.

Example 5. The double Laplace transform of the function $(\sin (x) / x)(\sin (t) / t)$, as follows:

$$
\begin{aligned}
& L_{x} L_{t}\left\{\frac{\sin (x)}{x} \frac{\sin (t)}{t} ; p, s\right\} \\
&=\left[D T _ { p } ^ { - 1 } D T _ { s } ^ { - 1 } \left\{D T _ { x } D T _ { t } \left\{\int_{y_{0}}^{y} \int_{\beta_{0}}^{\beta} \frac{\sin (x)}{x} \frac{\sin (t)}{t}\right.\right.\right. \\
& \\
&\left.\left.\left.\times e^{-p x} e^{-s t} d t d x\right\}\right\}\right]_{y_{0}=0, \beta_{0}=0}^{y=\infty, \beta=\infty} .
\end{aligned}
$$

By using sin series, we have

$$
\begin{aligned}
& \frac{\sin (x)}{x} \frac{\sin (t)}{t} \\
& =\left(\frac{\sum_{i=0}^{\infty}\left((-1)^{i} x^{2 i+1} /(2 i+1) !\right)}{x}\right) \\
& \quad \times\left(\frac{\sum_{j=0}^{\infty}\left((-1)^{j} t^{2 j+1} /(2 j+1) !\right)}{t}\right) \\
& =\left(\sum_{i=0}^{\infty} \frac{(-1)^{i} x^{2 i}}{(2 i+1) !}\right)\left(\sum_{j=0}^{\infty} \frac{(-1)^{j} t^{2 j}}{(2 j+1) !}\right) .
\end{aligned}
$$

By applying double differential transform, we have

$$
\begin{aligned}
D T_{x} D T_{t}\left\{\frac{\sin (x)}{x} \frac{\sin (t)}{t}\right\} \\
=\left\{\sum_{i=0}^{\infty} \frac{(-1)^{i}}{(2 i+1) !} D T_{x}\left\{x^{2 i}\right\}\right\} \\
\times\left\{\sum_{j=0}^{\infty} \frac{(-1)^{j}}{(2 j+1) !} D T_{t}\left\{t^{2 j}\right\}\right\} .
\end{aligned}
$$

On using double inverse differential transform, we have

$$
\begin{aligned}
L_{x} L_{t} & \left\{\frac{\sin (x)}{x} \frac{\sin (t)}{t} ; p, s\right\} \\
= & {\left[D T_{p}^{-1}\left\{\frac{(-1)^{m / 2}}{(m+1) !} \frac{(-p)^{k-m-1} /(k-m-1) !}{k}\right\}\right]_{y_{0}=0}^{y=\infty} } \\
& \times\left[D T_{s}^{-1}\left\{\frac{(-1)^{n / 2}}{(n+1) !} \frac{(-s)^{\beta-n-1} /(\beta-n-1) !}{\beta}\right\}\right]_{\beta_{0}=0}^{\beta=\infty} \\
= & {\left[\sum _ { k = 0 } ^ { \infty } \left\{\sum _ { m = 0 , 2 , 4 } ^ { k - 1 } \left\{\frac{(-1)^{m / 2}(k-1)(k-2) \cdots(k-m)}{(m+1) !(-p)^{m+1}}\right.\right.\right.} \\
\times & {\left[\sum _ { \beta = 0 } ^ { \infty } \left\{\sum _ { n = 0 , 2 , 4 } ^ { \beta - 1 } \left\{\frac{(-1)^{n / 2}(\beta-1)(\beta-2) \cdots(\beta-n)}{(n+1) !(-s)^{n+1}}\right.\right.\right.} \\
\left.\left.\left.\times(-1)^{m+1}\right\} \frac{(-p x)^{k}}{k !}\right\}\right]_{y_{0}=0}^{y=\infty} & \left.\left.\left.\times(-1)^{n+1}\right\} \frac{(-s t)^{\beta}}{\beta !}\right\}\right]_{\beta_{0}=0}^{\beta=\infty}
\end{aligned}
$$

$$
\begin{aligned}
& L_{x} L_{t}\left\{\frac{\sin (x)}{x} \frac{\sin (t)}{t} ; p, s\right\} \\
& =\left[\sum _ { k = 0 } ^ { \infty } \left\{\left(-\frac{1}{p}+\frac{(k-1)(k-2)}{3 ! p^{3}}\right.\right.\right. \\
& -\frac{(k-1)(k-2)(k-3)(k-4)}{5 ! p^{5}} \\
& +((k-1)(k-2)(k-3)(k-1) \\
& \left.\times(k-5)(k-6)) \times\left(7 ! p^{7}\right)^{-1}+\cdots\right) \\
& \left.\left.\times \frac{(-p x)^{k}}{k !}\right\}\right]_{y_{0}=0}^{y=\infty} \\
& \times\left[\sum _ { \beta = 0 } ^ { \infty } \left\{\left(-\frac{1}{s}+\frac{(\beta-1)(\beta-2)}{3 ! s^{3}}\right.\right.\right. \\
& -\frac{(\beta-1)(\beta-2)(\beta-3)(\beta-4)}{5 ! s^{5}} \\
& +((\beta-1)(\beta-2)(\beta-3)
\end{aligned}
$$




$$
\begin{aligned}
& \left.\times(\beta-1)(\beta-5)(\beta-6)) \times\left(7 ! s^{7}\right)^{-1}+\cdots\right) \\
& \left.\left.\times \frac{(-s t)^{\beta}}{\beta !}\right\}\right]_{\beta_{0}=0}^{\beta=\infty} .
\end{aligned}
$$

According to the previous lemma, we have

$$
\begin{aligned}
& L_{x} L_{t}\left\{\frac{\sin (x)}{x} \frac{\sin (t)}{t} ; p, s\right\} \\
& =\left[\sum _ { k = 0 } ^ { \infty } \left\{\left(-\frac{1}{p}+\frac{2 !}{3 ! p^{3}}-\frac{4 !}{5 ! p^{5}}+\frac{6 !}{7 ! p^{7}}+\cdots\right)\right.\right. \\
& \left.\left.\times \frac{(-p x)^{k}}{k !}\right\}\right]_{y_{0}=0}^{y=\infty} \\
& \times\left[\sum _ { \beta = 0 } ^ { \infty } \left\{\left(-\frac{1}{s}+\frac{2 !}{3 ! s^{3}}-\frac{4 !}{5 ! s^{5}}+\frac{6 !}{7 ! s^{7}}+\cdots\right)\right.\right. \\
& \left.\left.\times \frac{(-s t)^{\beta}}{\beta !}\right\}\right]_{\beta_{0}=0}^{\beta=\infty}, \\
& L_{x} L_{t}\left\{\frac{\sin (x)}{x} \frac{\sin (t)}{t} ; p, s\right\} \\
& =\left(-\frac{1}{p}+\frac{2 !}{3 ! p^{3}}-\frac{4 !}{5 ! p^{5}}+\frac{6 !}{7 ! p^{7}}+\cdots\right)\left[e^{-p x}\right]_{y_{0}=0}^{y=\infty} \\
& \times\left(-\frac{1}{s}+\frac{2 !}{3 ! s^{3}}-\frac{4 !}{5 ! s^{5}}+\frac{6 !}{7 ! s^{7}}+\cdots\right)\left[e^{-s t}\right]_{\beta_{0}=0}^{\beta=\infty}, \\
& L_{x} L_{t}\left\{\frac{\sin (x)}{x} \frac{\sin (t)}{t} ; p, s\right\} \\
& =\left(\frac{1}{p}-\frac{1}{3 p^{3}}+\frac{1}{5 p^{5}}-\frac{1}{7 p^{7}}+\cdots\right) \\
& \times\left(\frac{1}{s}-\frac{1}{3 s^{3}}+\frac{1}{5 s^{5}}-\frac{1}{7 s^{7}}+\cdots\right) .
\end{aligned}
$$

By using the definition of

$$
\int \frac{d x}{x^{2}+a 2}=\frac{1}{a} \arctan \left(\frac{x}{a}\right)
$$

we have

$$
\begin{aligned}
\arctan \left(\frac{1}{p}\right) \arctan \left(\frac{1}{s}\right) \\
=\left(\frac{1}{p}-\frac{1}{3 p^{3}}+\frac{1}{5 p^{5}}-\frac{1}{7 p^{7}}+\cdots\right) \\
\times\left(\frac{1}{s}-\frac{1}{3 s^{3}}+\frac{1}{5 s^{5}}-\frac{1}{7 s^{7}}+\cdots\right) .
\end{aligned}
$$

So that

$$
L_{x} L_{t}\left\{\frac{\sin (x)}{x} \frac{\sin (t)}{t} ; p, s\right\}=\arctan \left(\frac{1}{p}\right) \arctan \left(\frac{1}{s}\right) .
$$

Also, we can use the same idea to compute double Laplace transform for convolution function, single or double.

\section{Acknowledgment}

The author gratefully acknowledge, that this project was supported by King Saud University, Deanship of Scientific Research, College of Science Research Center.

\section{References}

[1] J. K. Zhou, Differential Transformation and Its Applications for Electrical Circuits, Huazhong University Press, Wuhan, China, 1986.

[2] C. K. Chen and S. H. Ho, "Solving partial differential equations by two-dimensional differential transform method," Applied Mathematics and Computation, vol. 106, no. 2-3, pp. 171-179, 1999.

[3] J. D. Cole, "On a quasi-linear parabolic equation occurring in aerodynamics," Quarterly of Applied Mathematics, vol. 9, pp. 225-236, 1951.

[4] F. Kangalgil and F. Ayaz, "Solitary wave solutions for the KdV and $\mathrm{mKdV}$ equations by differential transform method," Chaos, Solitons \& Fractals, vol. 41, no. 1, pp. 464-472, 2009.

[5] S. Momani, Z. Odibat, and I. Hashim, "Algorithms for nonlinear fractional partial differential equations: a selection of numerical methods," Topological Methods in Nonlinear Analysis, vol. 31, no. 2, pp. 211-226, 2008.

[6] A. Arikoglu and I. Ozkol, "Solution of fractional differential equations by using differential transform method," Chaos, Solitons \& Fractals, vol. 34, no. 5, pp. 1473-1481, 2007.

[7] Z. M. Odibat, "Differential transform method for solving Volterra integral equation with separable kernels," Mathematical and Computer Modelling, vol. 48, no. 7-8, pp. 1144-1149, 2008.

[8] A. Arikoglu and I. Ozkol, "Solution of difference equations by using differential transform method," Applied Mathematics and Computation, vol. 174, no. 2, pp. 1216-1228, 2006.

[9] H. Fatoorehchi and H. Abolghasemi, "Computation of analytical Laplace transforms by the differential transform method," Mathematical and Computer Modelling, vol. 56, no. 7-8, pp. 145151, 2012. 


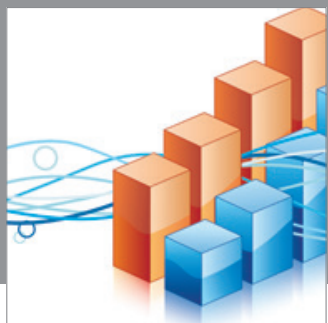

Advances in

Operations Research

mansans

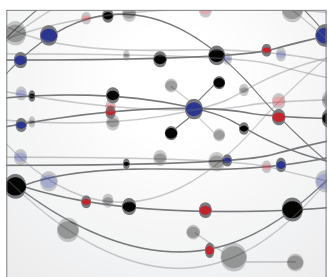

The Scientific World Journal
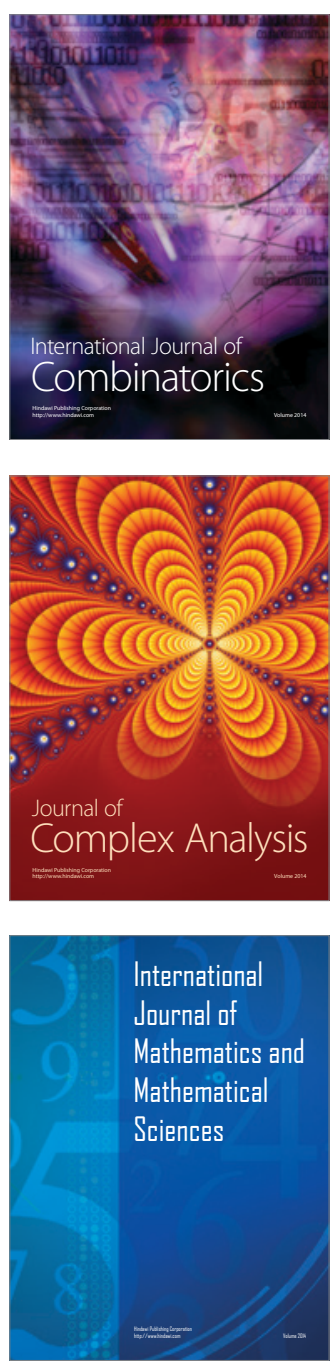
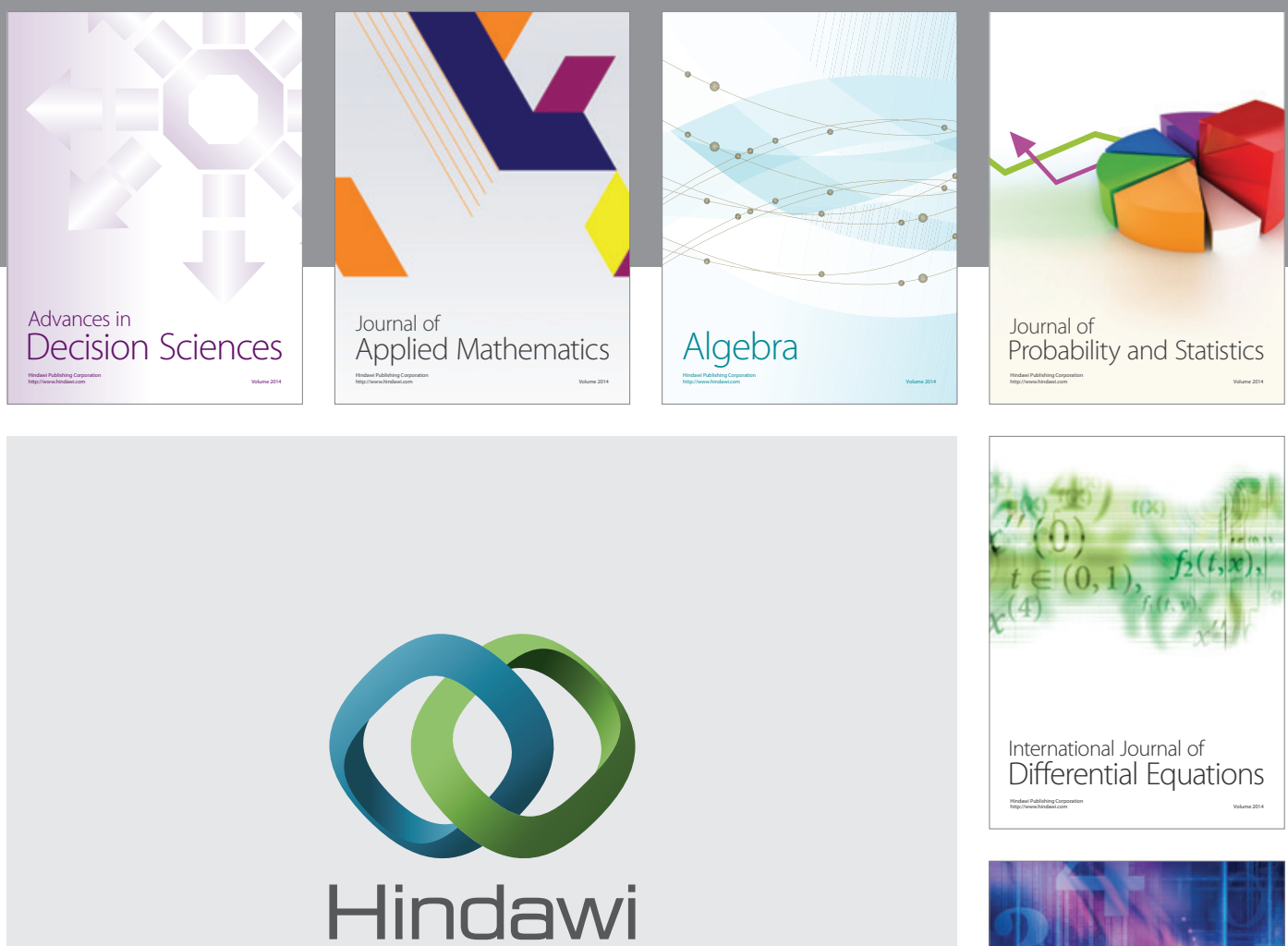

Submit your manuscripts at http://www.hindawi.com
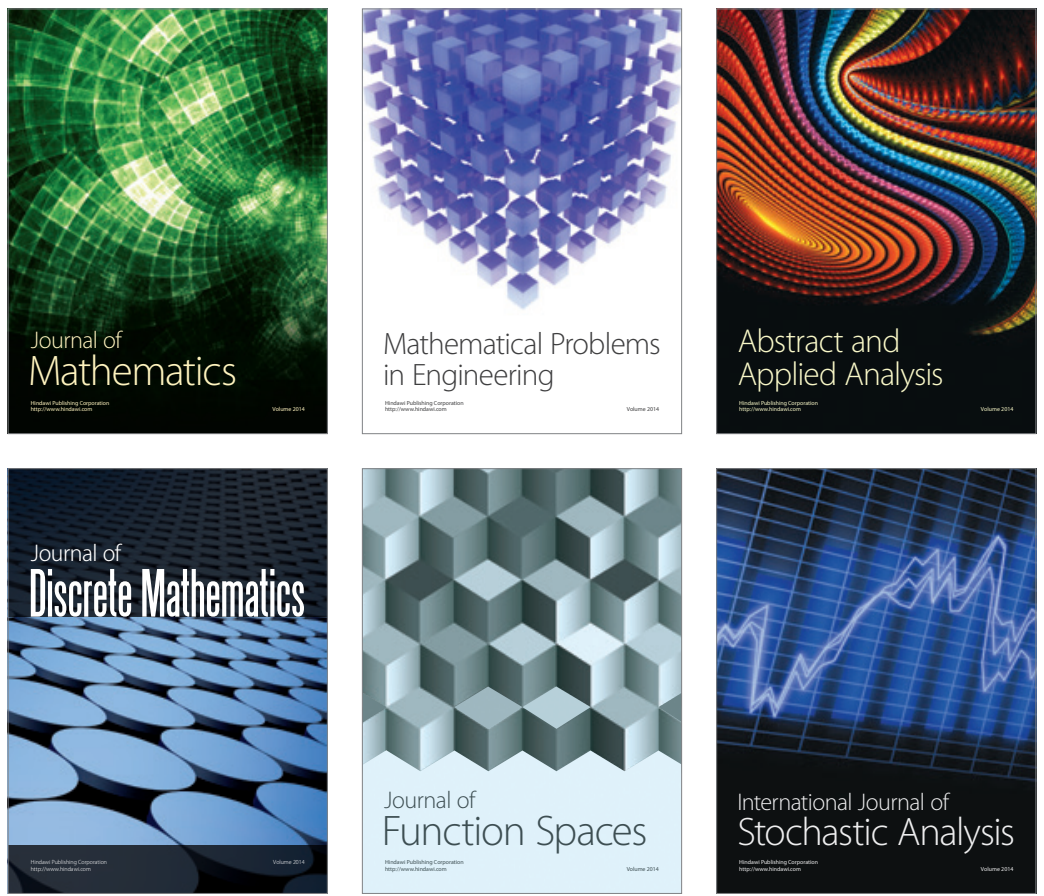

Journal of

Function Spaces

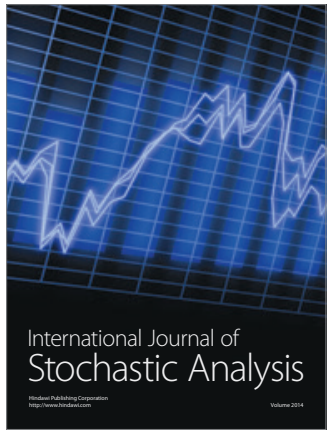

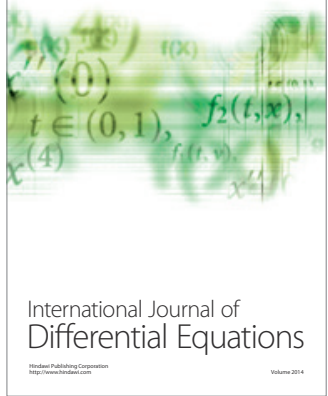
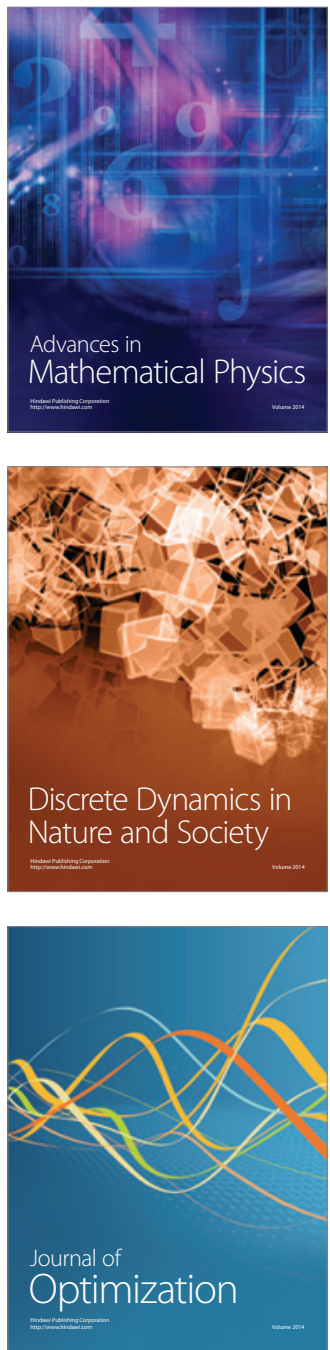